\title{
Survey on Spatial Item Recommender System using SPORE Model
}

\author{
Yuvraj Chougale $^{1}$, Prof. B.S. Satpute ${ }^{2}$ \\ Research Scholar, Dept. of Computer Science, DYP College of Engineering, Pimpri, Pune, India ${ }^{1}$ \\ Professor, Dept. of Computer Science, DYP College of Engineering, Pimpri, Pune, India ${ }^{2}$
}

\begin{abstract}
In recent year, Location based social network provide different user location features and recommendation. In location recommendation provide geographical influence and social influence with spatial item, and temporal data. A newly features stored user visit location. The user-item matrix is very sparse, which creates a big challenge for traditional collaborative filtering-based recommender systems. In Location based service has low sampling rate which existing prediction rate. In proposed system, we implement sequential personalized spatial item recommendation framework which provide user interest personal influence. We calculate the performance of SPORE on two datasets (Foursquare, Twitter) and one large-scale synthetic dataset. The results improvement SPORE ability to recommend spatial items, in terms of both efficiency and effectiveness, compared with the state-of-the-art methods.
\end{abstract}

Keywords: Location-based service, Location-based Social Network, Temporal data, spatial item.

\section{INTRODUCTION}

In recent year, rapid development in Web 2.0, mobile technologies, social networking and location-based social networks (LBSNs). Twitter and foursquare are provide the location based service [2], [7].

HuijiGao [4], In location based social networking site like twitter, facebook and foursquare are "check in" allow forget user location in your smart phones and share to our site for recommend new friends and circle. To aiming recommend new Points of Interest location to a user according to his personal preferences and facilitate his survey of new areas of the city.

In location recommendation system, user has recommend user which are visit in same place. Hongzhi Yin [9], has discuss two challenging question. 1. If we want to visit places in a city, where should we go? 2. If we want to join local occasions such as exhibitions and dramas in acuity, which events should we attend? We propose the notion of spatial items to denote both venues and events. User visit in limited places so it can easily store in browser history. Using collaborative filtering user send to recommendation.

In existing work, users' personal static check-in prediction through physical check-ins for location reference [4]. Inspired this features user can find similar places new friends. To find location based friend to need for improve location based social networking services. LBSN present unparalleled large-scale check-in data to label a user's mobile behaviour temporal and social aspects as well as spatial item.

In spatial item are found to user visiting places e.g. restaurant or cinema hall. JieBao [10], to developed location based preference recommendation system. In this system, send user visiting location list based on GPS with geospatial range. User are move in new city then it's not available in spatial list. As a user can only visit a limited number of locations, the user locations matrix is sparse, a big challenge to collaborative filtering-based location recommender systems.

\section{BACKGROUND}

In social website has added location based features which has find out user location through GPS [5], [7]. The system is stored user location in latitude and longitude format. In POI system [5], [6] user has get recommendation about existing places to a user according to his personal preferences and facilitate his exploration of new areas of the city [4].Inspired by POI developed location based social network system. In this system, display user update in social media.

A. Low Sampling Rate

In GPS and LBSN [6] has two location based features both functionality are same for getting user location [5].In the survey, access the datasets of Gowalla, twitter and LBSN. The LBSN data has low sampling compared to other in space and time. It is difficult to model the dependency between two check-in LBSNs using the location prediction techniques on GPS routes.

\section{B. Huge Prediction Space}

In LBSN [2], [7] has send to recommendation of user to which is your next places. In this system has stored the history or preferences of system which has check and send to recommendation. These preferences are stored in sequence format. If user visit to this location then check to collaborative filtering through recommendation. In system has called as Markov chains. They utilize sequential influence based on the first-order Markov chain for 
Vol. 5, Issue 12, December 2016

efficiency, but this only considers the newest visited place of a user to approximation the probability of user visiting a new location. A user visiting a new location through the developed additive Marko chain reflects the effect of all visited places in the check-in history of the user on the new location.

C. Unifying personalization and sequential effect

In recommendation systems using spatial item we have send to user personal visiting preferences which are stored in system. The system has stored user personal interest which has depends upon user frequently visiting places. In this visiting places has used to Markov chain in sequential format. If any user visits in places then send recommendation about predecessor or successor.

\section{III.RELATED WORK}

In this section, we discuss related work on location based social recommendations with spatial item. It has 4 existing approaches collaborative filtering, geographical influence, social influence, and sequential influence.

\section{A. Collaborative filtering:}

In existing recommendation techniques has used to point of interest system has collaborative filtering techniques on users' check-in data in GPS,LBSNs data and text data. The performance is limited for the GPS and LBSN system. This system limitation over to social influence, sequential influence, Social influence. Based on the statistic that nearby friends are more likely to share common interests, social link information has been widely applied to recover the value of recommender systems in LBSNs.

\section{B. Geographical influence:}

In Point of recommendation system has depends upon personal interest and geographical interest. In geographical interest user has visit any location then find out this related local preferences in system. The influence of geographical information of places on user check-in behaviours. These locations are used to user recommendation. The distance between two locations stayed by the same customer as a common delivery for all customers, a power-law delivery or a multi-centre Gaussian perfect. The geographical effect by exposing a personalized non-parametric transfer for each consumer.

\section{Sequential influence}

Location based service stored a user activity as preferences as sequentially. In these sequential influences has different sequence format store. Like different location stored in sequential format. In some case has different user visit from same places in different way. So system confusing to stored sequence and recommendation time.
Foursquare: Foursquare holds the check-in history of 4 , 163 records. Who worked in USA and Californiain Dec 2009and Jul 2013? Each check-in activity contains the user ID, item ID, item location, item content and a checkin time.

Twitter: This dataset is publicly available. Twitter dataset in which does not contain the category and tag information about spatial items. Twitter supports third party location sharing services like Foursquare and Gowalla and Foursquare dataset.

Synthetic Dataset. To find online reference datasets available using foursquare dataset. There are 4,163 record, 121,142 spatial items and 483,813 check-ins on the foursquare dataset.

\section{CONCLUSION}

In location based recommendation system has to incrementally mine sequential influence, in sequential influence on users' check inactivity in LBSNs. This system a user with spatial item references within the inquiring city based on the individual interests and the local favourites mined from the user's action past. To developed SPORE framework introduces a latent variable topic-region to model and fuse the personal interests and sequential influence in the latent space.

\section{REFERENCES}

[1]. H. Yin, B. Cui, L. Chen, Z. Hu, and Z. Huang, "A temporal context aware model for user behaviour modeling in social media systems," in SIGMOD, 2014, pp. 1543-1554.

[2]. J. Bao, Y. Zheng, and M. F. Mokbel, "Location-based and preference aware recommendation using sparse geo-social networking data," in SIGSPATIAL, 2012, pp. 199-208.

[3]. D. Lian, C. Zhao, X. Xie, G. Sun, E. Chen, and Y. Rui, "Geomf: Joint geographical modeling and matrix factorization for point-ofinterest recommendation," in KDD, 2014, pp. 831-840. H. Gao, J. Tang, X. Hu, and H. Liu, "Exploring temporal effects for location recommendation on location-based social networks," in RecSys, 2013, pp. 93-100.

[4]. J.-D. Zhang and C.-Y. Chow, "Spatiotemporal sequential influence modelling for location recommendations: A gravity-based approach," TIST, 2015.

[5]. H. Yin, B. Cui, X. Zhou, W. Wang, Z. Huang, and S. Sadiq, "Joint modelling of user check-in behaviours for real-time point-ofinterest recommendation," TOIS, 2016.

[6]. H.-P. Hsieh, C.-T. Li, and S.-D. Lin, "Measuring and recommending time-sensitive routes from location-based data," TIST, vol. 5, no. 3, pp. 45:1-45:27, 2014.

[7]. J.-D. Zhang, C.-Y. Chow, and Y. Li, "Lore: Exploiting sequential influence for location recommendations," in SIGSPATIAL, 2014, pp. 103-112.

[8]. H. Yin, Y. Sun, B. Cui, Z. Hu, and L. Chen, "Lcars: A locationcontent aware recommender system," in KDD, 2013, pp. 221-229.

[9]. J. Bao, Y. Zheng, and M. F. Mokbel, "Location-based and preference aware recommendation using sparse geo-social networking data," in SIGSPATIAL, 2012, pp. 199-208.

\section{IV.DATASET}

In our survey we have found two datasets i.e. Foursquare and one large synthetic, Twitter. 